\title{
Dysfunction of Fronto-Subcortical Circuitry in Fronto-Temporal Dementia
}

\author{
S. Laurentino ${ }^{1}$, E. B. Sougey ${ }^{2,3}$ \\ ${ }^{1}$ Clinic of Behavioral Neurology and Neurophysiology, Recife, Brazil \\ ${ }^{2}$ Post Graduate Program of Neuropsychiatry and Behavioral Sciences, Federal University of Pernambuco, Recife, Brazil \\ ${ }^{3}$ Department of Neuropsychiatry, Federal University of Pernambuco, Recife, Brazil \\ Email: silvialaurentino@gmail.com
}

How to cite this paper: Laurentino, S. and Sougey, E.B. (2020) Dysfunction of Fronto-Subcortical Circuitry in Fronto-Temporal Dementia. Open Journal of Psychiatry, 10, 171-186.

https://doi.org/10.4236/ojpsych.2020.104015

Received: May 18, 2020

Accepted: August 24, 2020

Published: August 27, 2020

Copyright (c) 2020 by author(s) and Scientific Research Publishing Inc. This work is licensed under the Creative Commons Attribution International License (CC BY 4.0).

http://creativecommons.org/licenses/by/4.0/ (c) (i) Open Access

\begin{abstract}
In the last years, some studies have shown that behavior disorder seems in frontotemporal dementia is related to dysfunction in the fronto-subcortical circuitry. Objectives: We did a narrative literature review concerning fronto-subcortical circuitry and frontotemporal dementia (FTD). Methods: Manuscripts related to fronto-subcortical network and frontotemporal dementia were selected for further analysis. Results: From the executions of simple motor actions to the most complex behaviors like goal-direct behavior and social cognition, the fronto-subcortical circuitry involves an intrigued network of fibers that reaches to basal ganglia nuclei. Recently, researchers have shown five parallel fronto-subcortical circuits integrating and segregating information from the frontal cortex to basal ganglia. Understanding the relationship between the fronto-subcortical circuit dysfunctions and neurodegenerative diseases requires studying the functional anatomy and neurochemical basis involved. Conclusions: In this view, it is essential to review the functional anatomy of the fronto-subcortical network, and it's correlated with clinical aspects to pursuing a better therapeutic approach.
\end{abstract}

\section{Keywords}

Frontotemporal Dementia, Fronto-Subcortical Circuitry, Basal Ganglia, Neurotransmitters, Fronto-Subcortical Syndrome

\section{Introduction}

An enormous explosion of life diversity occurred in the Cambrian era; vertebrates like fish, amphibians, reptiles, birds, and mammals developed a vertebrate skeleton, which allowed them to have more fast movement. This highly sophis- 
ticated and advanced nervous system was related to the high degree of encephalization. This evolution of life diversity occurred around 620 - 590 million years ago [1]. With the myelination of the nervous system, some animals improved the speed velocity of neural transmission, creating a high ability to transmit neuronal signals in a saltatory way to increase the temporal precision and promote faster communication between the brain and the body [1] [2] [3].

Between 85 mya and six mya, the pre-humans appeared in the life history. The diet that was frugivorous at the beginning of evolution became rich in iodine and essential fatty acid with a seafood introduction. This new diet promotes a boosting of dopamine activity and intellectual development, which played a factor in advancing cerebral connectivity, ultimately making us modern humans [1] [4]-[10]. More than just an increase in brain volume, the key to our specie evolution was morphological brain changes and connectivity changes that reorganized the frontal and prefrontal cortex, besides other multimodal associative cortex [1] [11] [12].

Some studies have shown that one of the most critical brain regions involve with human behavior is the prefrontal cortex (PFC) [13] [14]. The PFC is subdivided into smaller architectonic areas based on their distinct neuronal organization. These include the number and size of the cortical layers, the size, shape, and density of the neurons and the degree of axon myelination [15]. An important finding was describing showing that the Brodmann's area 10 in the human brain was larger than the rest of the brain, and the supragranular layers have more space available for connections with other higher-order association areas [15]. This finding suggested that the neural substrates supporting cognitive functions associated with this part of the cortex enlarged and became specialized during hominid evolution [15].

In summary, we should say that the frontal lobe works as a "hub" integrating different cortical functions to flexible goal-directed behavior and adaptive response [16]. More recently, researches have shown the existence of five parallel fronto-subcortical circuits integrating and segregating information from the frontal cortex to basal ganglia [17]. These circuits included the supplementary motor area, frontal eye field, dorsolateral prefrontal region, lateral orbitofrontal region, and the cingulate portion of the prefrontal cortex, which in turn, forms loops from the cortical areas to the striatum, pallidal complex nuclei, and thalamus, then returning to the cortex [17] [18]. It is important to emphasize that a specific architecture and multiple neurotransmitter interactions modulate each circuit's functional activity, and any dysfunction can cause behavioral disorders. In this view, it is essential to correlate functional connectivity, neurochemical pathway, and clinical aspects to pursue a better therapeutic approach for the treatment of fronto-subcortical syndromes related to the behavioral variant of frontotemporal dementia (bv-FTD).

\section{The Fronto-Subcortical Circuits}

The fronto-subcortical circuitry related to behavioral function will operate to 
modulate drive behavior, motivation, and executive function to better organize strategy, motor planning, and execution for adequate decision-making [16]-[22]. Three parallel segregated circuits reach the dorsal and ventral striatum, Globus pallidus, substantia nigra and thalamus, and modulate motivation, reward, and emotions response [16] [17] [18] [20] [21] [23] [24]. The dorsolateral prefrontal (DLPFC) and associate parietal cortex (APC) will modulate the executive functions while the anterior cingulate cortex (ACC) will participating on the motivation and reward system and, finally the orbitofrontal lateral cortex will modulate the behavior inhibitory control (Figure 1).

\section{The Frontostriatal Connectivity and Synaptic Plasticity}

The striatum is the main input structure of basal ganglia formed for three major sources from the cerebral cortex, thalamus, and brainstem [16] [19] [26] [27] [28]. As the basal ganglia's primary input nucleus, the striatum will participate to receive, integrate, or segregate from other incoming signals and relayed to appropriate outputs [29]. The striatum also can be subdivided into the dorsal and ventral striatum. The dorsal striatum nucleus is dividing into the dorsomedial (DMS) and dorsolateral (DLS) regions, receiving projections from the frontal and parietal-associated cortex and sensorimotor cortex. On the other hand, the ventral striatum/nuclei Accumbens (NAc) receives projections from limbic structures, including the amygdala, hippocampus, medial prefrontal cortex, and anterior cingulate cortex [16] [26] [29] [30] [31]. This basal nucleus also receives indirect cortical input through the intralaminar thalamic nuclei, especially the centromedian-parafascicular nuclei (CM-pf).

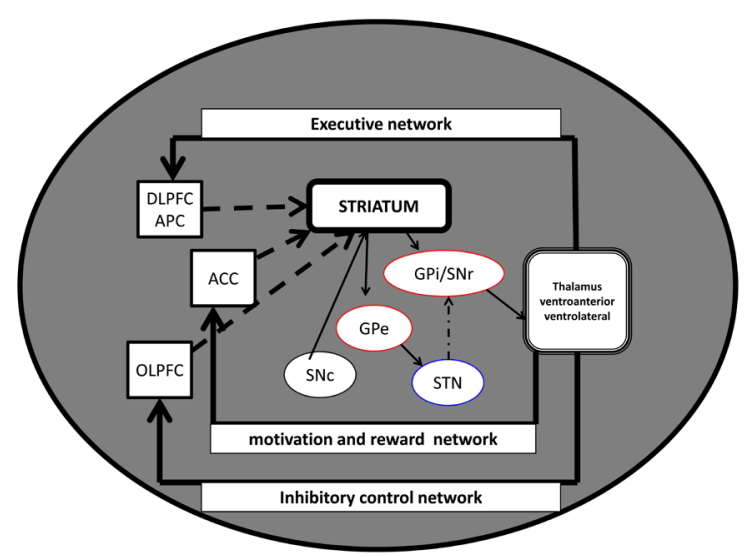

Figure 1. Simplified Fronto-subcortical pathways. DLPFC $=$ dorsolateral prefrontal cortex; ACC = anterior cingulate cortex; OLPFC = orbitofrontal lateral prefrontal cortex; Striatum; $\mathrm{GPi}=$ globus pallidus internus $; \mathrm{SNr}=$ substantia nigra reticulata; $\mathrm{GPe}=$ globus pallidus externus; $\mathrm{SNc}=$ substantia nigra compacta; $\mathrm{STN}=$ subthalamic nuclei, $\mathrm{APC}=$ associative parietal cortex. Adapted from: David G. Lichter \& J. L Cummings. Introduction and overview. In: Frontal-Subcortical Circuits in Psychiatric and Neurological Disorders. 1 ed. New York NGP, editor. United States of America: The Guilford Press; 2001 2000. 448 p. [25]; Tekin et al., 2002 ( Tekin S, Cummings JL). Frontal-subcortical neuronal circuits and clinical neuropsychiatry: an update. J Psychosom Res. 2002; 53(2): 647-54) [18]. 
The striatum contains several cell types that are generally divided into two general groups: projection neurons and interneurons [16] [32] [33]. Most cells are GABAergic neurons, including a large population of medium spiny neurons (MSNs) and interneurons. Furthermore, it's essential to address a lack of striatal glutamatergic neurons, although many cortical glutamatergic neurons are projecting to the striatum [16] [24]. The GABAergic's striatal interneurons can be divided into at least two classes, based on their physiological properties: 1) fastspiking (FS-parvalbumin-positive cells) and 2) Low-threshold spiking (somatostatin-oxide-synthase, and neuropeptide-Y-positive cells (NPY); also potentially calretinin-positive interneurons) [32] [34]. Although interneurons constitute a tiny population of the dorsal striatum compared to that of MSNs, the minority exerts powerful inhibitory effects on MSNs through GABAergic transmission. There is also a dense innervation of mesencephalic dopaminergic axons that reaches the striatum and the presence of cholinergic interneurons [35].

Besides the GABAergic interneurons, there are two classes of striatal dopaminergic receptors: D1-like (D1 and D5 receptors) and D2-like (D2, D3, and D4 receptors). All dopaminergic receptors are coupled with G-protein receptors. However, D1 receptors will activate Gs proteins, while D2 stimulates inhibitory Gi proteins [35]. The D1 receptor (D1R) stimulates the adenylciclase and presenting a phasic response to dopamine. On the other hand, the D2 receptor will respond in the tonic way to inhibit the neurons' activity through the Gi-coupled signaling pathway [20] [26] [36].

It is also essential to address that the substantia nigra compacta $(\mathrm{SNc})$, which project dopaminergic neurons to the striatum, will form clusters called cell islands [16]. These cell islands are histochemically organized in two compartments: 1) striossomes, and 2) matrix. Striossomes occupy 10\% - 15\% of striatal volume and are rich in substance $\mathrm{P}(\mathrm{SP})$, which acts increasing DA release and has a vital role in the direct pathway, which express MSNs D1R. The matrix, by contrast, is enriched with enkephalin (ENK), D2-receptor (D2R), and acetylcholine and cholinergic markers, including acetylcholine esterase (AChE) and choline acetyltransferase (ChAT) and have an essential role in the indirect pathway [29].

In summary, once the glutamatergic inputs from the sensorimotor cortex reach the striatum and activate the direct-pathway, MSNs D1 receptors will project directly to GABAergic neurons in the GPi and $\mathrm{SNr}$, activating these neurons, which in turn sending axons to motor nuclei of the thalamus, become inhibited [35]. This information flow's net effect, which promote a disinhibition of excitatory thalamocortical projections [16] [24] [26] [29] [35] [37] [38] [39] [40].

Furthermore, the indirect pathway also receives glutamatergic inputs from the cortex. The glutamatergic input onto GABAergic MSNs D2 receptors inhibits GABAergic pallidal neurons of the GPe. In this case, the target of the GPe neurons, the glutamatergic neurons of the subthalamic nuclei (STN), are disinhibited [35]. Finally, the disinhibition of STN glutamatergic neurons could activate 
inhibitory output neurons of the GPi and SNr, resulting in an inhibition of excitatory thalamocortical projection neurons promoting a reduction of excitatory projections from the thalamus to the cortex, inhibiting the motor movement [35].

Another mesencephalic area crucial in the fronto-subcortical circuits is the ventral tegmental area (VTA). Projections from VTA can be classified as mesolimbic and mesocortical. An essential mesolimbic projection of the VTA is the nucleus Accumbens (NAc), olfactory tubercle, amygdala, and septum. The mesocortical pathway reach the prefrontal, cingulate, and perirhinal cortex [41] [42] besides locus ceruleus and other cortical areas. One of the most critical roles of the mesocorticolimbic pathway is to work in goal-direct behavior, drug induced-reward, selective attention, and working memory [43].

\section{Others Neurotransmitters Systems Influencing Fronto-Subcortical Circuits}

One of the most important neurotransmitters of the mammalian brain is the norepinephrine (NE). These neurotransmitters modulated attention, arousal, and cognition during many behaviors and works associated with dopamine (DA). It's shown that noradrenergic neurons from the locus ceruleus project to the entire cortex and hippocampus, cerebellum, and spinal cord. In the cerebral cortex, B1 receptors receive noradrenergic neurons and works associated with DA to promote a better signal-to-noise ratio of the atentional system. Studies showed that blocking NE transporters leads to an increase in DA and NE levels in the prefrontal cortex [44]. These findings suggest that the DA action in the frontal cortex is modulating by NE transporters [44] [45] [46].

Furthermore, there is a prominent innervation of serotonin neurons and an essential expression of 5HT receptors distributed in the prefrontal cortex modulating part of the cortical function [43] [47] [48]. Some 5HT receptors can cause frontal inhibition on the DA neurons [48] [49]. For instance, 5HT2c and 5HT2a decrease DA in the frontal cortex and striatum, while the 5HT3, 5HT1a, and $5 \mathrm{HT} 1 \mathrm{~b}$ increase the release of DA in the frontal cortex, striatum, amygdala and hippocampus [48] [50].

One of the essential neurochemical modulation related to a balance of behavior control is the interaction between the DA and 5HT receptors (5HT/DA interactions) both in the basal ganglia and frontal cortex [43] [51]. The cell bodies and terminal regions of all three DA pathways (SNc, mesolimbic and mesocortical) are innervated by 5 -HT neurons originating in the medial and dorsal raphe nuclei [43] [47] [52] [53] [54] [55]. Thus, 5-HT could potentially regulate the function of DA neurons via actions on midbrain DA cell bodies and DA terminals [43].

\section{Clinical Aspects of the Fronto-Subcortical Behavioral Syndromes}

One of the most relevant clinical aspects of frontotemporal dementia is a beha- 
vior disorder. Impaired executive functions, apathy, and impulsivity are hallmarks of frontal-subcortical circuit dysfunction [23] [56] [57]. Exist three forms of behavioral variant Frontotemporal dementia (bv-FTD): 1) dorsolateral prefrontal syndrome (dysexecutive syndrome) which the patients have severe impairment in planning and executed some tasks related to attention and selecting goals; 2) anterior cingulate syndrome (apathetic syndrome) characterized by apathy with a deficit in complex motor control and, lack in error detection; 3) lateral orbitofrontal syndrome (disinhibition syndrome) which have a hard control in terms of therapeutic response due to a severe imbalance related to emotional reaction leading to mania or hypomania episodes, lack of judgment and social clues and, inappropriate sexual conduct leading to problematic social life (Figure 2) [18] [23] [37] [40] [58] [59].

\subsection{The Dorsolateral Prefrontal Syndrome}

The dorsolateral prefrontal cortex (DLPC) organized all aspects of executive functions. This circuit originates in BA 9 and BA10 [23] [37]. Fibers from these regions send glutamatergic excitatory projections to the head of caudate dorsolateral. Caudate nuclei, in turn, send inhibitory GABAergic projections to the lateral aspect of the mediodorsal GPi and rostrolateral $\mathrm{SNr}$ via the direct pathway, which express MSNs D1 receptors. Once GPi is inhibited, it won't send inhibitory GABAergic projections to mediodorsal thalamus, which, in turn, becomes disinhibited and increases excitatory predictions back to the DLPC closing the disinhibitory looping [18] [19] [23] [31] [35] [39] [58] [59]. On the other hand, the indirect pathway, which expresses MSNs D2 receptors, will send GABAergic inhibitory projections to dorsal GPe, which in turn project GABAergic

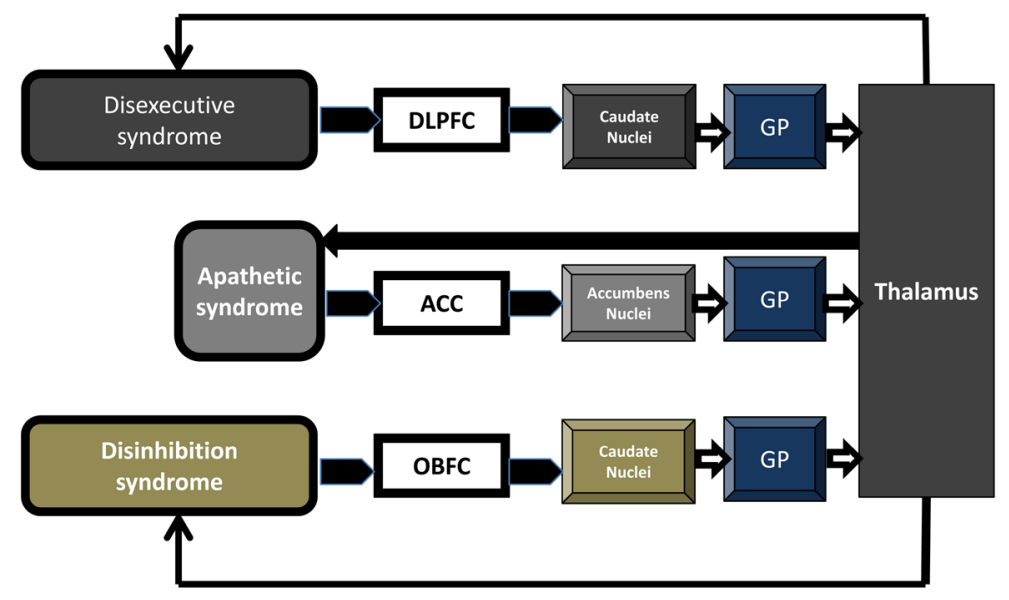

Figure 2. Fronto-subcortical syndromes. DLPFC—dorsolateral prefrontal cortex, ACCanterior cingulate cortex, OBFC-orbitofrontal córtex, GP-globus Pallidus. Adapted from: David G. Lichter \& J. L Cummings. Introduction and overview. In: Frontal-Subcortical Circuits in Psychiatric and Neurological Disorders. 1 ed. New York NGP, editor. United States of America: The Guilford Press; 2001 2000. 448 p: 59-87 [25]; Tekin et al., 2002 (Tekin S, Cummings JL). Frontal-subcortical neuronal circuits and clinical neuropsychiatry. an update. J Psychosom Res. 2002; 53(2): 647-54) [18]. 
neurons to subthalamic nuclei, and after that will send Glutamatergic projections to GPi. In this case, the GPi increases the GABAergic inhibitory projections to thalamus, decreasing the glutamatergic projections to the DLPC, making BA9 and BA10 inhibited [18] [19] [23] [31] [35] [39] [58] [59] [60] (Figure 3).

The dysexecutive syndrome is characterized by dysfunction in the system that regulates the executive plans and monitors errors to adjust task performance [18]. Patients with dysexecutive-FTD have difficulty maintaining attention, shifting sets to control the mistakes in response to changing task demands, and selecting goals for a better decision making related to social contingencies [18]. Also, there are associated features like reduced verbal and design fluency, impairment of memory search strategies on learning, and motor programming disturbances [25]. This dysexecutive syndrome also can see in other neuropsychiatric disorders like Parkinson's disease, Attention Deficit Disorders, Depression, etc. [18].

\subsection{The Anterior Cingulate Prefrontal Syndrome}

The anterior cingulate cortex (ACC) has two subdivisions: 1) the dorsal cognitive division (BA 32, 24); and 2) rostro-ventral affective division or limbic cortex [25] [61]. The cognitive division of ACC works as an atentional network interconnected with the dorsolateral prefrontal cortex (BA 46/9), parietal cortex (BA 7); premotor, and supplementary motor area. This area will be connected with caudate nuclei sending projections to $\mathrm{GPi} / \mathrm{SNr}$ and thence to the mediodorsal

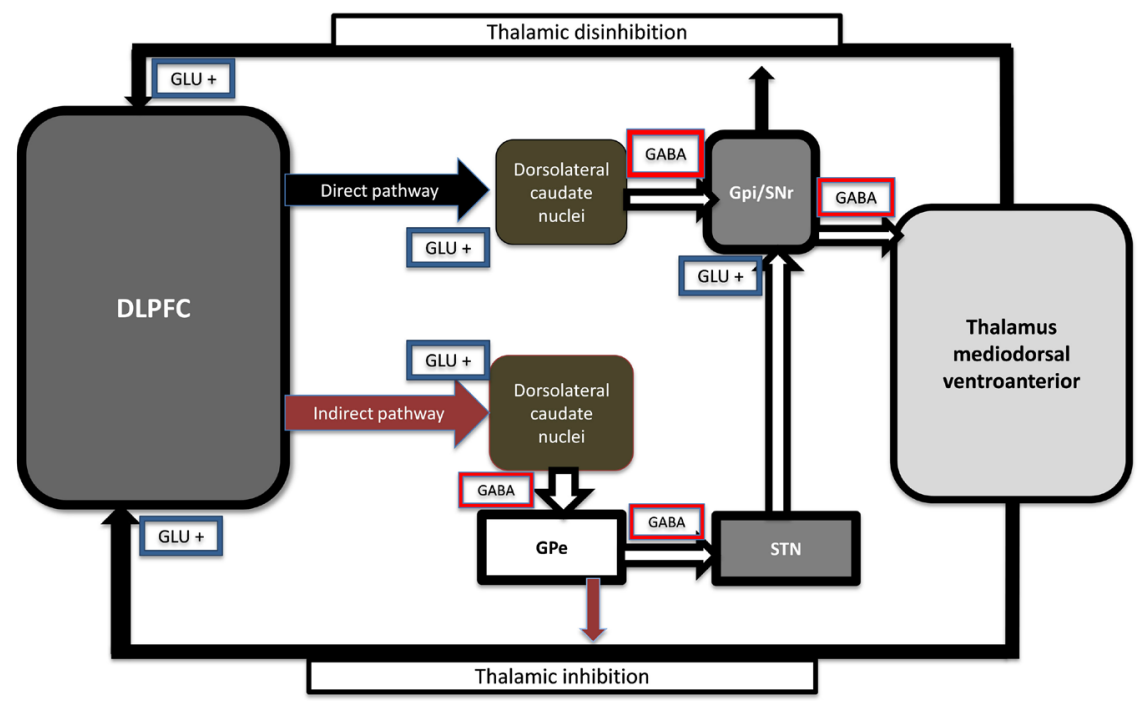

Figure 3. Dorsolateral circuitry. Glut+: glutamate excitation; GABA: GABA inhibition, Gpi-globus Pallidus internus, GPe-globo Pallidus externus, SNr-substantia Nigra reticulata, STN—-subthalamic nuclei. Adapted from: David G. Lichter \& J.L Cummings. Introduction and overview. In: Frontal-Subcortical Circuits in Psychiatric and Neurological Disorders. 1 ed. New York NGP, editor. United States of America: The Guilford Press; 2001 2000. 448 p; 2001: 59-87 [25]; Tekin et al., 2002 (Tekin S, Cummings JL). Frontal-subcortical neuronal circuits and clinical neuropsychiatry: an update. J Psychosom Res. 2002; 53(2): 647-54) [18]. 
thalamus nuclei. The thalamic nuclei, especially the mediodorsal and ventral anterior portion, and the intralaminar nuclei will close the looping sending projections back to the dorsal division of ACC turn, succeeding projection to DLPC [27] [61]. This integrated network links the dorsal ACC with the dorsolateral cortex. It will be responsible for modulating executive or attention functions, especially motivational behavior, complex motor control, error detection, anticipating cognitive task, and working memory [25] [61] (Figure 4).

The second division of ACC originates from Brodmann's area 24 and is part of the ventral striatum (VS) [58]. The ventral striatum includes the ventromedial caudate, ventral putamen, nucleus Accumbens, and olfactory tubercle [58] [62]. The VS sends projections to innervate the rostromedial GPi and ventral Pallidum (VP) and the rostrodorsal STN [63]. There may also be a less well-defined indirect loop projecting from ventral striatum to the rostral pole of the GPe [64]. The external pallidum, in turn, connects to the medial STN, which returns projections to the ventral pallidum [65] and, then to the mediodorsal thalamus [66]. Finally, the looping is closed when the mediodorsal thalamus sends the final projection back to the limbic cortex, reaching the rostromedial portion [24] (Figure 5). It is essential to address that the ventral subdivision of ACC comprises the subgenual cingulate division (ACCsg) (BA 32), which predominantly connects with the amygdala, medial orbitofrontal cortex, anterior insula, medial temporal lobe, hypothalamus, periaqueductal grey and dorsal brainstem [13] [61] [67]. This circuit is related to salience monitoring of emotional and motivational information link the salience social stimulus with affective processing and inhibitory control [13] [20] [22] [24] [62] [64] [67] [68]. Dysfunction of the ACC circuit produces akinetic mutism characterized by profound apathy, lack of spontaneous verbalization and movement [13] [18] [23] [58] [67] [69] [70].

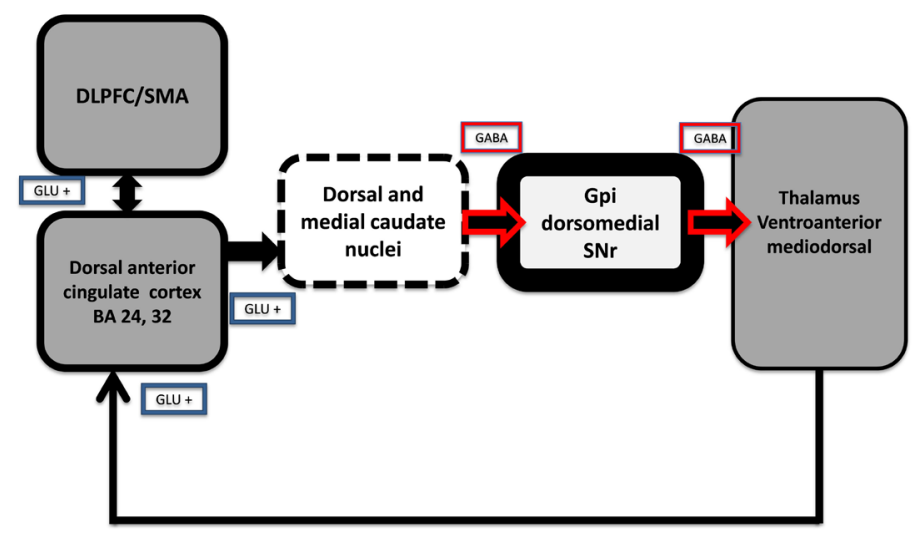

Figure 4. Cognitive anterior cingulate cortex circuitury. Glut+: glutamate excitation; GABA-: GABA inhibition, GP-globus Pallidus, SNr-substantia Nigra reticulata. Adapted from: David G. Lichter \& J. L Cummings. Introduction and overview. In: Frontal-Subcortical Circuits in Psychiatric and Neurological Disorders. 1 ed. New York NGP, editor. United States of America: The Guilford Press; 2001 2000. 448 p; 2001: $59-87$ [25]; Tekin et al., 2002 (Tekin S, Cummings JL). Frontal-subcortical neuronal circuits and clinical neuropsychiatry: an update. J Psychosom Res. 2002; 53(2): 647-54) [18]. 


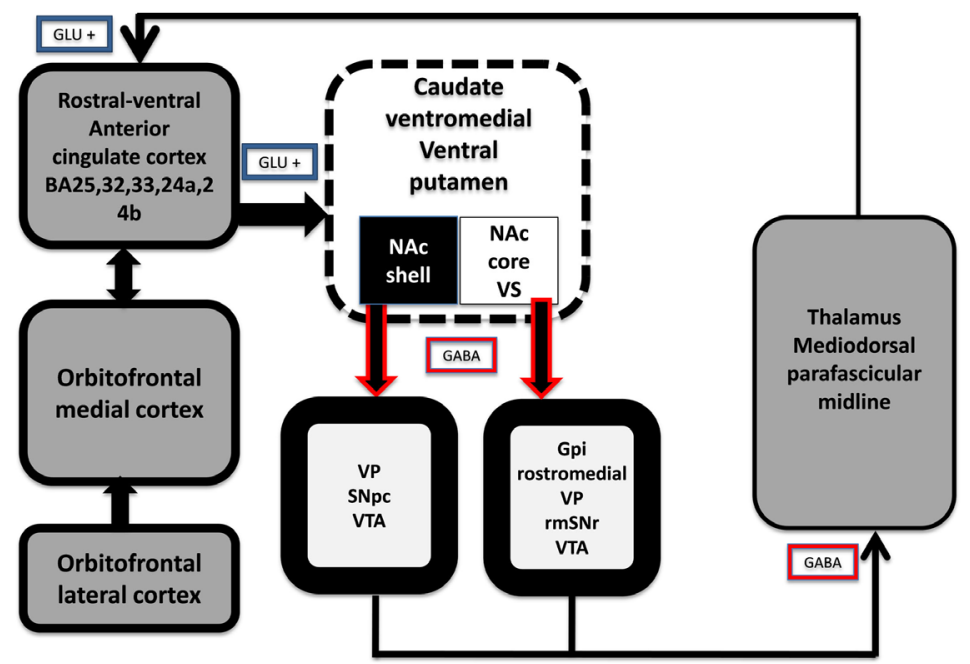

Figure 5. Rostro-ventral affective anterior cingulate cortex circuitury. Glut+: glutamate excitation; GABA-: GABA inhibition, DLPFC-NAc-nucleus Accumbens, VP-ventral pallidum, $\mathrm{SNpc}$ - substantia nigra pars compacta, $\mathrm{rmSNr}$ - rostromedial substantia nigra reticulata, GPi-globus Pallidus internus, VTA-ventral tegmental area. Adapted from: David G. Lichter \& J.L Cummings. Introduction and overview. In: Frontal-Subcortical Circuits in Psychiatric and Neurological Disorders. 1 ed. New York NGP, editor. United States of America: The Guilford Press; 2001 2000. 448 p; 2001: 59-87 [25]; Tekin et al., 2002 (Tekin S, Cummings JL). Frontal-subcortical neuronal circuits and clinical neuropsychiatry: an update. J Psychosom Res. 2002; 53(2): 647-54) [18].

\subsection{The Orbitofrontal Syndrome}

The orbitofrontal cortex also shows projections towards the basal ganglia. Brodmann's area 11 corresponds to the orbitofrontal circuit, and BA 10 and 47 corresponds to the medial part of the frontal gyrus. Both will send projections to the basal ganglia, especially the ventromedial part of the caudate nuclei. This area also receives projections from the visual cortex, auditory association cortex, and upper and lower temporal gyrus [17] [18] [23] [58].

The ventromedial caudate nuclei, in turn, send GABAergic projections to the medial segment of GPi, which is located medially to the dorsolateral caudate nuclei and the rostromedial portion of the SNr [17]. The GPi, in turn, sends GABAergic projections straightforward to the ventral-anterior and dorsomedial thalamic nucleus [17] [58] [71]. The thalamic nucleus will send back glutamatergic projections to the lateral orbitofrontal cortex, closing the direct inhibitory circuit [18] [58] [71]. There is also an indirect pathway that project GABAergic neurons out of the ventromedial nuclei to the GPe, which will send GABAergic projections to the lateral subthalamic nuclei (LSTN). The LSTN, in turn, sends glutamatergic projections to GPi and SNr. The GPi and SNr neurons will project onto the thalamus in the medial magnocellular part of the ventral-anterior nucleus. In the magnocellular inferomedial portion of the mediodorsal thalamus, closing the OLPFC inhibitory circuit (Figure 6) [58].

The orbitofrontal circuit provides an essential role in the control of emotional behavior. Thus, lesions in this area disconnect the frontal monitoring system 


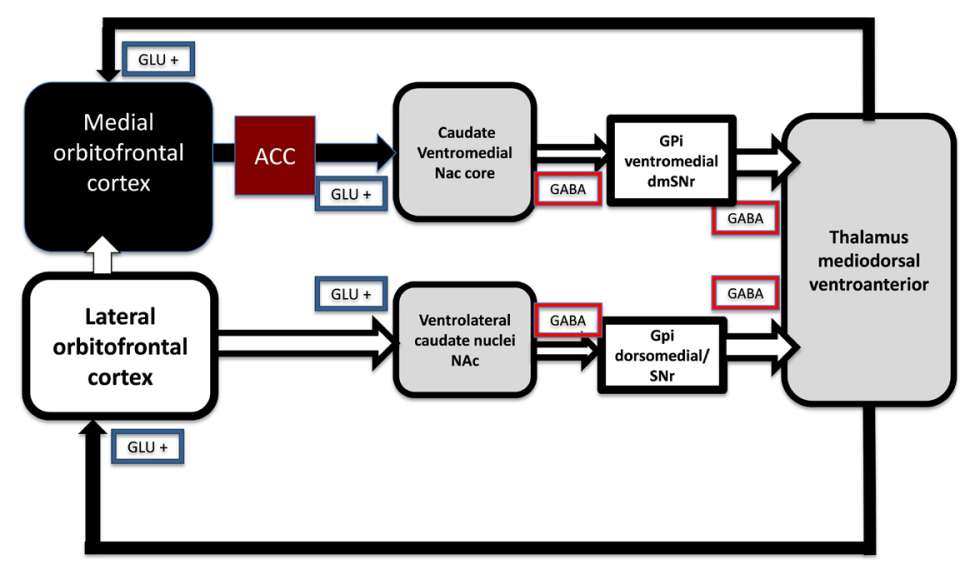

Figura 6. Medial and lateral orbitofrontal circuitry. Glut+: glutamate excitation; GABA-: GABA inhibition, ACC-anterior cingulate cortex, $\mathrm{dmSNr}$ - dorsomedial substantia nigra reticulata, Gpi-globus Pallidus internus, NAc-nucleus Accumbens, SNr-substantia nigra reticulata. Adapted from: David G. Lichter \& J.L Cummings. Introduction and overview. In: Frontal-Subcortical Circuits in Psychiatric and Neurological Disorders. 1 ed. New York NGP, editor. United States of America: The Guilford Press; 2001 2000. 448 p; 2001: 59-87 [25]; Tekin et al., 2002 (Tekin S, Cummings JL). Frontal-subcortical neuronal circuits and clinical neuropsychiatry: an update. J Psychosom Res. 2002; 53(2): 647-54) [18].

from limbic input control (70). Another significant dysfunction of OFC syndrome is impulsive and disinhibition disorder, which affects their social life in different ways where patients have a lack of judgment and social clues, inappropriate sexual remarks, antisocial behavior as irritability, emotional lability, mania or hypomania, abnormal motor behavior [37] [58] [72] [73] [74] [75].

\section{Final Considerations}

In the last years, some researches have shown the link between fronto-subcortical circuits dysfunction and neuropsychiatric disease. The fronto-subcortical circuit dysfunction can be treated with some drugs that acting modulating neurotransmitters in this pathway. It's imperative to consider that understanding the functional anatomy and neurochemical modulation related to this network is the best way to choose drugs based on the comprehension of their action in the fronto-subcortical pathway. For instance, to understand to balance between serotonin receptors and dopamine release in the frontal lobe, or the effect of NE receptors in the Dopa release on the frontal lobe, as well as, the importance of mesocortical, mesolimbic and mesostriatal dopamine pathway will contribute to choose a better therapeutic approach in front of the challenge, which is to control some behavioral disorders seems in the behavioral variant of frontotemporal dementia (bv-FTD).

\section{Conflicts of Interest}

The authors declare no conflicts of interest regarding the publication of this paper. 


\section{References}

[1] Hoffmann, M. (2013) The Human Frontal Lobes and Frontal Network Systems: An Evolutionary, Clinical, and Treatment Perspective. ISRN Neurology, 2013, Article ID: 892459. https://doi.org/10.1155/2013/892459

[2] Zalc, B. (2006) The Acquisition of Myelin: A Success Story. Novartis Foundation Symposium, 276, 15-54, 275-281. https://doi.org/10.1002/9780470032244.ch3

[3] Zalc, B., Goujet, D. and Colman, D. (2008) The Origin of the Myelination Program in Vertebrates. Current Biology, 18, R511-R512. https://doi.org/10.1016/j.cub.2008.04.010

[4] Holloway Jr., R.L. (1968) The Evolution of the Primate Brain: Some Aspects of Quantitative Relations. Brain Research, 7, 121-172.

https://doi.org/10.1016/0006-8993(68)90094-2

[5] Holloway, R.L. (1983) Cerebral Brain Endocast Pattern of Australopithecus afarensis Hominid. Nature, 303, 420-422. https://doi.org/10.1038/303420a0

[6] Holloway, R.L. and De La Costelareymondie, M.C. (1982) Brain Endocast Asymmetry in Pongids and Hominids: Some Preliminary Findings on the Paleontology of Cerebral Dominance. American Journal of Physical Anthropology, 58, 101-110. https://doi.org/10.1002/ajpa.1330580111

[7] Coppens, Y. (1988) The Vicissitudes of Human Evolution. Bulletin de P Académie Nationale de Médecine, 172, 1289-1296.

[8] Coppens, Y. (1991) The Anatomical and Functional Origin of the First Bipedalism. Bulletin de I Académie Nationale de Médecine, 175, 977-991.

[9] Coppens, Y. (1994) East Side Story: The Origin of Humankind. Sci Am, 270, 88-95. https://doi.org/10.1038/scientificamerican0594-88

[10] Coppens, Y. (1998) The Evolution of Man. Bulletin et Memoires de PAcademie Royale de Medecine de Belgique, 153, 447-449.

[11] Raghanti, M.A., et al. (2008) Cortical Dopaminergic Innervation among Humans, Chimpanzees, and Macaque Monkeys: A Comparative Study. Neuroscience, 155, 203-220. https://doi.org/10.1016/j.neuroscience.2008.05.008

[12] Barger, N., Stefanacci, L. and Semendeferi, K. (2007) A Comparative Volumetric Analysis of the Amygdaloid Complex and Basolateral Division in the Human and Ape Brain. American Journal of Physical Anthropology, 134, 392-403. https://doi.org/10.1002/ajpa.20684

[13] Rudebeck, P.H., et al. (2008) Frontal Cortex Subregions Play Distinct Roles in Choices between Actions and Stimuli. Journal of Neuroscience, 28, 13775-13785. https://doi.org/10.1523/JNEUROSCI.3541-08.2008

[14] Mesulam, M.M. (1986) Frontal Cortex and Behavior. Annals of Neurology, 19, 320-325. https://doi.org/10.1002/ana.410190403

[15] Semendeferi, K., et al. (2001) Prefrontal Cortex in Humans and Apes: A Comparative Study of Area 10. American Journal of Physical Anthropology, 114, 224-241. https://doi.org/10.1002/1096-8644(200103)114:3\%3C224::AID-AJPA1022\%3E3.0.C $\underline{\mathrm{O} ; 2-\mathrm{I}}$

[16] Haber, S.N. (2016) Corticostriatal Circuitry. In: Pfaff, D. and Volkow, N., Eds., Neuroscience in the 21 st Century, Springer, New York, 7-21.

https://doi.org/10.1007/978-1-4614-6434-1 135-1

[17] Alexander, G.E., Delong, M.R. and Strick, P.L. (1986) Parallel Organization of Functionally Segregated Circuits Linking Basal Ganglia and Cortex. Annual Review of Neuroscience, 9, 357-381. https://doi.org/10.1146/annurev.ne.09.030186.002041 
[18] Tekin, S. and Cummings, J.L. (2002) Frontal-Subcortical Neuronal Circuits and Clinical Neuropsychiatry: An Update. Journal of Psychosomatic Research, 53, 647-654. https://doi.org/10.1016/S0022-3999(02)00428-2

[19] Heilbronner, S.R. and Haber, S.N. (2014) Frontal Cortical and Subcortical Projections Provide a Basis for Segmenting the Cingulum Bundle: Implications for Neuroimaging and Psychiatric Disorders. Journal of Neuroscience, 34, 10041-10054. https://doi.org/10.1523/JNEUROSCI.5459-13.2014

[20] Morris, L.S., et al. (2016) Fronto-Striatal Organization: Defining Functional and Microstructural Substrates of Behavioural Flexibility. Cortex, 74, 118-133. https://doi.org/10.1016/j.cortex.2015.11.004

[21] Postuma, R.B. and Dagher, A. (2006) Basal Ganglia Functional Connectivity Based on a Meta-Analysis of 126 Positron Emission Tomography and Functional Magnetic Resonance Imaging Publications. Cerebral Cortex, 16, 1508-1521. https://doi.org/10.1093/cercor/bhj088

[22] Masterman, D.L. and Cummings, J.L. (1997) Frontal-Subcortical Circuits: The Anatomic Basis of Executive, Social and Motivated Behaviors. Journal of Psychopharmacology, 11, 107-114. https://doi.org/10.1177/026988119701100203

[23] Cummings, J.L. (1993) Frontal-Subcortical Circuits and Human Behavior. Archives of Neurology, 50, 873-880. https://doi.org/10.1001/archneur.1993.00540080076020

[24] Alexander, G.E. and Crutcher, M.D. (1990) Functional Architecture of Basal Ganglia Circuits: Neural Substrates of Parallel Processing. Trends in Neurosciences, 13, 266-271. https://doi.org/10.1016/0166-2236(90)90107-L

[25] Lichter, D.G. and Cummings, J.L. (2001) Introduction and Overview. In: FrontalSubcortical Circuits in Psychiatric and Neurological Disorders, The Guilford Press, New York, 448 p.

[26] Macpherson, T. and Hikida, T. (2019) Role of Basal Ganglia Neurocircuitry in the Pathology of Psychiatric Disorders. Psychiatry and Clinical Neurosciences, 73, 289-301. https://doi.org/10.1111/pcn.12830

[27] Delong, M.R., et al. (1984) Role of Basal Ganglia in Limb Movements. Human Neurobiology, 2, 235-244.

[28] Haber, S.N. (2011) Neuroanatomy of Reward: A View from the Ventral Striatum. In: Gottfried, J.A., Ed., Neurobiology of Sensation and Reward, CRC Press, Boca Raton.

[29] Brimblecombe, K.R. and Cragg, S.J. (2017) The Striosome and Matrix Compartments of the Striatum: A Path through the Labyrinth from Neurochemistry toward Function. ACS Chemical Neuroscience, 8, 235-242. https://doi.org/10.1021/acschemneuro.6b00333

[30] Monchi, O., Ko, J.H. and Strafella, A.P. (2006) Striatal Dopamine Release during Performance of Executive Functions: A [ $\left.{ }^{11} \mathrm{C}\right]$ Raclopride PET Study. Neurolmage, 33, 907-912. https://doi.org/10.1016/j.neuroimage.2006.06.058

[31] Sesack, S.R. and Grace, A.A. (2010) Cortico-Basal Ganglia Reward Network: Microcircuitry. Neuropsychopharmacology, 35, 27-47. https://doi.org/10.1038/npp.2009.93

[32] Kreitzer, A.C. and Malenka, R.C. (2008) Striatal Plasticity and Basal Ganglia Circuit Function. Neuron, 60, 543-554. https://doi.org/10.1016/j.neuron.2008.11.005

[33] Haber, S.N. (2014) The Place of Dopamine in the Cortico-Basal Ganglia Circuit. Neuroscience, 282, 248-257. https://doi.org/10.1016/j.neuroscience.2014.10.008

[34] Kawaguchi, Y., et al. (1995) Striatal Interneurones: Chemical, Physiological and 
Morphological Characterization. Trends in Neurosciences, 18, 527-535. https://doi.org/10.1016/0166-2236(95)98374-8

[35] Do, J., et al. (2012) Functional Roles of Neurotransmitters and Neuromodulators in the Dorsal Striatum. Learning Memory, 20, 21-28.

https://doi.org/10.1101/lm.025015.111

[36] Surmeier, D.J., Song, W.J. and Yan, Z. (1996) Coordinated Expression of Dopamine Receptors in Neostriatal Medium Spiny Neurons. Journal of Neuroscience, 16, 6579-6591. https://doi.org/10.1523/JNEUROSCI.16-20-06579.1996

[37] Mega, M.S. and Cummings, J.L. (1994) Frontal-Subcortical Circuits and Neuropsychiatric Disorders. The Journal of Neuropsychiatry and Clinical Neurosciences, 6, 358-370. https://doi.org/10.1176/jnp.6.4.358

[38] Monchi, O., et al. (2006) Functional Role of the Basal Ganglia in the Planning and Execution of Actions. Annals of Neurology, 59, 257-264.

[39] Ikemoto, S., Yang, C. and Tan, A. (2015) Basal Ganglia Circuit Loops, Dopamine and Motivation: A Review and Enquiry. Behavioural Brain Research, 290, 17-31. https://doi.org/10.1016/j.bbr.2015.04.018

[40] Delong, M.R. and Wichmann, T. (2007) Circuits and Circuit Disorders of the Basal Ganglia. Archives of Neurology, 64, 20-24. https://doi.org/10.1001/archneur.64.1.20

[41] Beier, K.T., et al. (2015) Circuit Architecture of VTA Dopamine Neurons Revealed by Systematic Input-Output Mapping. Cell, 162, 622-634.

https://doi.org/10.1016/j.cell.2015.07.015

[42] Bourdy, R., et al. (2014) Control of the Nigrostriatal Dopamine Neuron Activity and Motor Function by the Tail of the Ventral Tegmental Area. Neuropsychopharmacology, 39, 2788-2798. https://doi.org/10.1038/npp.2014.129

[43] Alex, K.D. and Pehek, E.A. (2007) Pharmacologic Mechanisms of Serotonergic Regulation of Dopamine Neurotransmission. Pharmacology \& Therapeutics, 113, 296-320. https://doi.org/10.1016/j.pharmthera.2006.08.004

[44] Stahl, S.M. (2003) Neurotransmission of Cognition, Part 3. Mechanism of Action of Selective NRIs: Both Dopamine and Norepinephrine Increase in Prefrontal Cortex. Journal of Clinical Psychiatry, 64, 230-231. https://doi.org/10.4088/JCP.v64n0301

[45] Stahl, S.M. (2003) Neurotransmission of Cognition, Part 2. Selective Nris Are Smart Drugs: Exploiting Regionally Selective Actions on Both Dopamine and Norepinephrine to Enhance Cognition. Journal of Clinical Psychiatry, 64, 110-111. https://doi.org/10.4088/JCP.v64n0201

[46] Stahl, S.M. (2003) Neurotransmission of Cognition, Part 1, Dopamine Is a Hitchhiker in Frontal Cortex: Norepinephrine Transporters Regulate Dopamine. Journal of Clinical Psychiatry, 64, 4-5. https://doi.org/10.4088/JCP.v64n0101

[47] Celada, P., Puig, M.V. and Artigas, F. (2013) Serotonin Modulation of Cortical Neurons and Networks. Frontiers in Integrative Neuroscience, 7, 25. https://doi.org/10.3389/fnint.2013.00025

[48] Puig, M.V. and Gulledge, A.T. (2011) Serotonin and Prefrontal Cortex Function: Neurons, Networks, and Circuits. Molecular Neurobiology, 44, 449-464. https://doi.org/10.1007/s12035-011-8214-0

[49] Benarroch, E.E. (2009) The Locus Ceruleus Norepinephrine System: Functional Organization and Potential Clinical Significance. Neurology, 73, 1699-1704. https://doi.org/10.1212/WNL.0b013e3181c2937c

[50] Hoyer, D. and Schoeffter, P. (1991) 5-HT Receptors: Subtypes and Second Messengers. Journal of Receptor Research, 11, 197-214. 
https://doi.org/10.3109/10799899109066399

[51] Coccaro, E.F. (1989) Central Serotonin and Impulsive Aggression. British Journal of Psychiatry, 155, 52-62. https://doi.org/10.1192/S0007125000291769

[52] Alex, K.D., et al. (2005) Modulation of Dopamine Release by Striatal 5-HT2C Receptors. Synapse, 55, 242-251. https://doi.org/10.1002/syn.20109

[53] Celada, P., Bortolozzi, A. and Artigas, F. (2013) Serotonin 5-HT $\mathrm{HT}_{1 \mathrm{~A}}$ Receptors as Targets for Agents to Treat Psychiatric Disorders: Rationale and Current Status of Research. CNS Drugs, 27, 703-716. https://doi.org/10.1007/s40263-013-0071-0

[54] Celada, P., et al. (2013) Disruption of Thalamocortical Activity in Schizophrenia Models: Relevance to Antipsychotic Drug Action. International Journal of Neuropsychopharmacology, 16, 2145-2163. https://doi.org/10.1017/S1461145713000643

[55] Palacios, J.M., et al. (1990) Distribution of Serotonin Receptors. Annals of the New York Academy of Sciences, 600, 36-52. https://doi.org/10.1111/j.1749-6632.1990.tb16871.x

[56] Cummings, J.L., et al. (1996) Neuropsychiatric Syndromes in Neurodegenerative Disease: Frequency and Significance. Seminars in Clinical Neuropsychiatry, 1, 241-247.

[57] Cummings, J.L. and Hegarty, A. (1994) Neurology, Psychiatry, and Neuropsychiatry. Neurology, 44, 209-213. https://doi.org/10.1212/WNL.44.2.209

[58] Bonelli, R.M. and Cummings, J.L. (2007) Frontal-Subcortical Circuitry and Behavior. Dialogues in Clinical Neuroscience, 9, 141-151.

[59] Haber, S.N. and Rauch, S.L. (2010) Neurocircuitry: A Window into the Networks Underlying Neuropsychiatric Disease. Neuropsychopharmacology, 35, 1-3.

[60] Kupchik, Y.M., et al. (2015) Coding the Direct/Indirect Pathways by D1 and D2 Receptors Is Not Valid for Accumbens Projections. Nature Neuroscience, 18, 12301232. https://doi.org/10.1038/nn.4068

[61] Bush, G., Luu, P. and Posner, M.I. (2000) Cognitive and Emotional Influences in Anterior Cingulate Cortex. Trends in Cognitive Sciences, 4, 215-222. https://doi.org/10.1016/S1364-6613(00)01483-2

[62] Chikama, M., et al. (1997) Insular Cortical Projections to Functional Regions of the Striatum Correlate with Cortical Cytoarchitectonic Organization in the Primate. Journal of Neuroscience, 17, 9686-9705. https://doi.org/10.1523/JNEUROSCI.17-24-09686.1997

[63] Critchley, H.D. (2005) Neural Mechanisms of Autonomic, Affective, and Cognitive Integration. Journal of Comparative Neurology, 493, 154-166. https://doi.org/10.1002/cne.20749

[64] Cauda, F., et al. (2011) Functional Connectivity and Coactivation of the Nucleus Accumbens: A Combined Functional Connectivity and Structure-Based MetaAnalysis. Journal of Cognitive Neuroscience, 23, 2864-2877. https://doi.org/10.1162/jocn.2011.21624

[65] Temel, Y., et al. (2005) The Functional Role of the Subthalamic Nucleus in Cognitive and Limbic Circuits. Progress in Neurobiology, 76, 393-413. https://doi.org/10.1016/j.pneurobio.2005.09.005

[66] Smith, Y. and Parent, A. (1988) Neurons of the Subthalamic Nucleus in Primates Display Glutamate but Not GABA Immunoreactivity. Brain Research, 453, 353-356. https://doi.org/10.1016/0006-8993(88)90177-1

[67] Rudebeck, P.H., Bannerman, D.M. and Rushworth, M.F. (2008) The Contribution of Distinct Subregions of the Ventromedial Frontal Cortex to Emotion, Social Be- 
havior, and Decision Making. Cognitive, Affective, \& Behavioral Neuroscience, 8, 485-497. https://doi.org/10.3758/CABN.8.4.485

[68] Benarroch, E.E. (2019) Insular Cortex: Functional Complexity and Clinical Correlations. Neurology, 93, 932-938. https://doi.org/10.1212/WNL.0000000000008525

[69] Eslinger, P.J. and Damasio, A.R. (1985) Severe Disturbance of Higher Cognition after Bilateral Frontal Lobe Ablation: Patient EVR. Neurology, 35, 1731-1741. https://doi.org/10.1212/WNL.35.12.1731

[70] Eslinger, P.J., et al. (2012) Apathy in Frontotemporal Dementia: Behavioral and Neuroimaging Correlates. Behavioural Neurology, 25, Article ID: 286427. https://doi.org/10.1155/2012/286427

[71] Ilinsky, I.A., Jouandet, M.L. and Goldman-Rakic, P.S. (1985) Organization of the Nigrothalamocortical System in the Rhesus Monkey. Journal of Comparative Neurology, 236, 315-330. https://doi.org/10.1002/cne.902360304

[72] Christidi, F., et al. (2018) Social Cognition Dysfunctions in Neurodegenerative Diseases: Neuroanatomical Correlates and Clinical Implications. Behavioural Neurology, 2018, Article ID: 1849794. https://doi.org/10.1155/2018/1849794

[73] Couture, S.M., Penn, D.L. and Roberts, D.L. (2006) The Functional Significance of Social Cognition in Schizophrenia: A Review. Schizophrenia Bulletin, 32, S44-S63.

[74] Kennedy, D.P. and Adolphs, R. (2012) The Social Brain in Psychiatric and Neurological Disorders. Trends in Cognitive Science, 16, 559-572.

https://doi.org/10.1016/j.tics.2012.09.006

[75] Shany-Ur, T. and Rankin, K.P. (2011) Personality and Social Cognition in Neurodegenerative Disease. Current Opinion in Neurology, 24, 550-555.

https://doi.org/10.1097/WCO.0b013e32834cd42a 


\section{Abbreviation Note List}

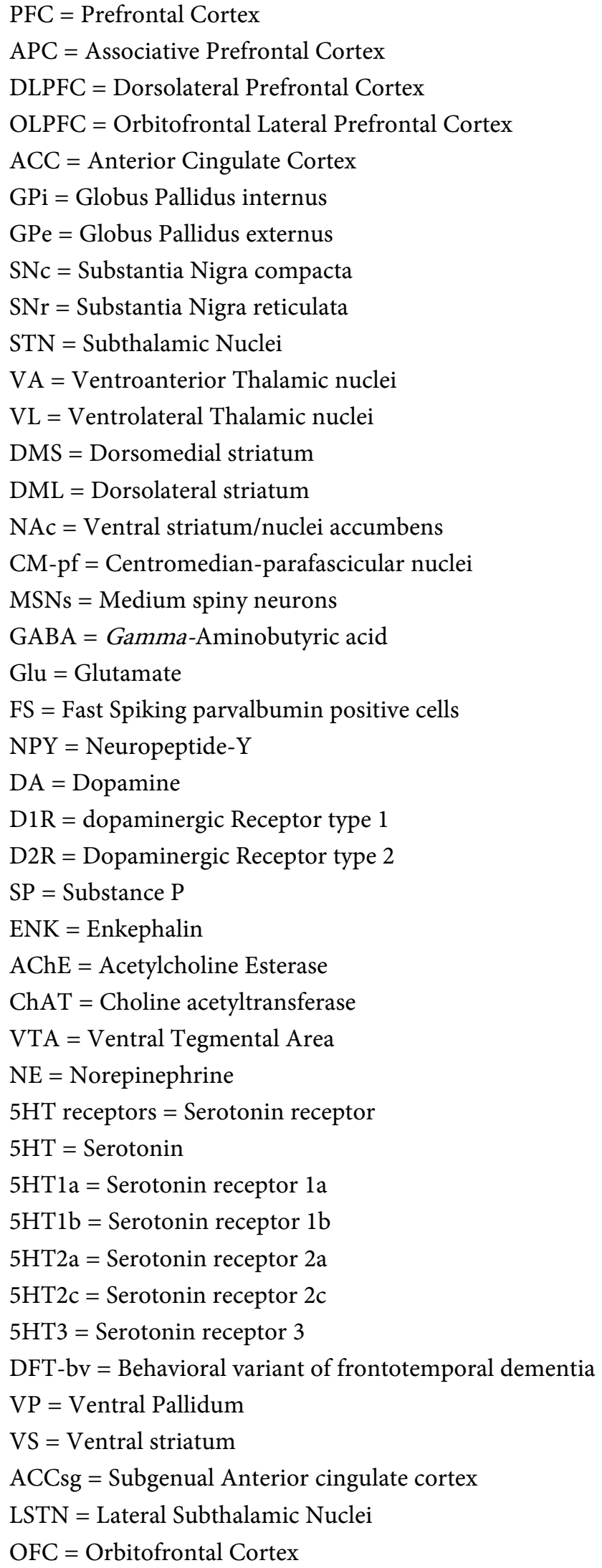

